Samantha S. Castaneda, MD ${ }^{1,2,3}$

Daniel M. Alonzo, MD'

Rodney Marc H. Ramos, MD

'Department of Otolaryngology Head and Neck Surgery The Medical City

${ }^{2}$ Department of Otorhinolaryngology

Head and Neck Surgery

Jose R. Reyes Memorial Medical Center

${ }^{3}$ Department of Otolaryngology Head and Neck Surgery Rizal Medical Center
Correspondence: Samantha S. Castaneda, MD Department of Otolaryngology Head and Neck Surgery The Medical City

Ortigas Avenue, Pasig City 1600

Phone: (632) 6356789 loc.6250 to 6251

Telefax: (632) 6873349

Email: docsamcastaneda@yahoo.com

Reprints will not be available from the author.

No funding support was received for this study. The authors signed a disclosure that they have no proprietary or financial interest with any organization that may have a direct interest in the subject matter of this manuscript, or in any product used or cited in this article.

\section{Anterolateral Thigh Flap Reconstruction of Full Thickness Buccal Defect}

\author{
ABSTRACT \\ Objective: To present our experience with the anterolateral thigh flap in reconstructing a full \\ thickness defect of the buccal mucosa and cheek.

\section{Methods:} \\ Design: Case Report \\ Setting: Tertiary Private Hospital \\ Patient: One
}

Results: A 36-year-old male with a T4aNOM0 Stage IVa buccal carcinoma on the left underwent wide excision, marginal mandibulectomy and modified radical neck dissection with preservation of the spinal accessory nerve, internal jugular vein and sternocleidomastoid muscle. The resultant through-and-through defect of the cheek skin measuring $8 \times 6 \mathrm{~cm}$ and buccal mucosa measuring $6 \times 10 \mathrm{~cm}$ with a concomitant $1 / 4$ upper lip and 1/3 lower lip defect was reconstructed with an anterolateral thigh free flap. The patient recovered uneventfully and underwent adjuvant concurrent chemoradiotherapy 1 month post-operation. At $2 \frac{1}{2}$ months post-operation, he had no oral incontinence and could resume a normal diet with good speech.

Conclusion: The anterolateral thigh free flap is an excellent soft-tissue flap for reconstruction of a full thickness defect of the buccal area.

Key words: Surgical flap; Carcinoma, squamous cell; Oral cancer

Resection of advanced head and neck malignancies can lead to extensive defects resulting in functional and aesthetic problems. When oncological resection is tailored to the reconstructive options available, margins of resection may be compromised. The introduction of microvascular free tissue transfers has broadened the options in head and neck reconstruction. The anterolateral thigh (ALT) flap is a fasciocutaneous flap of the thigh that has not been as popular a choice as the radial forearm flap due to the variable anatomy of the vascular pedicle and the relatively difficult technique of dissection. ${ }^{1}$

In the Philippine setting, there has been no reported case of reconstruction using the ALT flap. We present our experience with the anterolateral thigh flap in reconstructing a through and through defect of the buccal mucosa and cheek. 


\section{CASE REPORTS}

\section{CASE REPORT}

A 36-year-old male was admitted for a T4aNOMO Stage IVa buccal squamous cell carcinoma. Physical examination centered on the oral cavity revealing a $5 \times 5$ centimeter fungating left buccal mucosal mass extending to the cheek and lip (Figure 1). Computed tomography scans from the zygoma to the clavicle with contrast showed erosion of the left posterior maxillary bone and hemimandible, and enlarged level 1 lymph nodes.
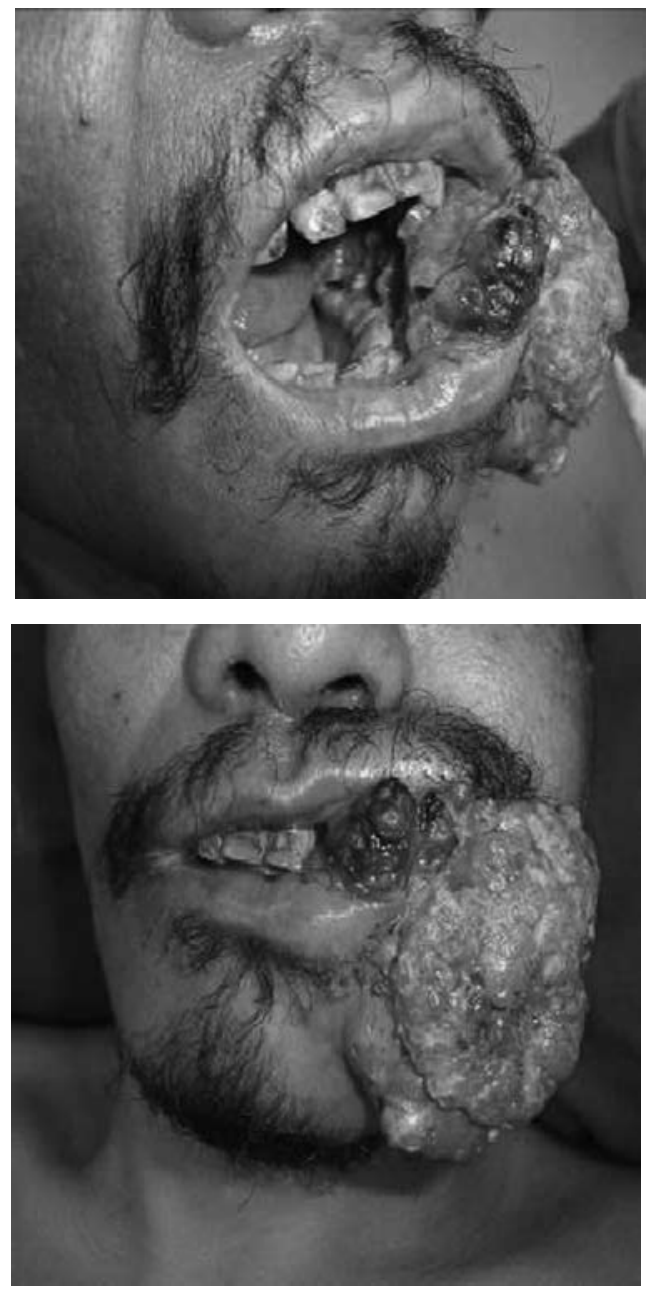

Figure 1. The tumor involving the cheek, lip and buccal mucosa.

Following a multidisciplinary meeting to determine the best possible management, the patient underwent wide excision with frozen section of the margins. The resultant buccal mucosa defect measured $10 \times 6$ $\mathrm{cm}$. One fourth of the upper lip and $1 / 3$ of the lower lip was excised. The cheek skin defect measured $8 \times 6 \mathrm{~cm}$. Marginal mandibulectomy from the $3^{\text {rd }}$ molar to the canine (Figure 2) and modified radical neck dissection with preservation of the sternocleidomastoid muscle, internal jugular vein and spinal accessory nerve were also performed.

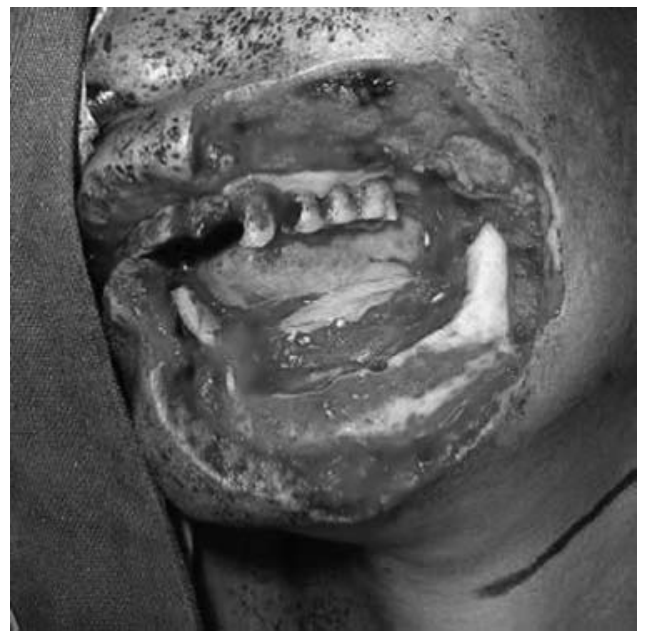

Figure 2. Extent of defect after resection

Simultaneous with the neck dissection, a separate team harvested the anterolateral thigh flap after determining the dimension of the defect. A $23 \times 9 \mathrm{~cm}$ flap was harvested based on a musculocutaneous perforator of the descending branch of the lateral circumflex femoral artery. The anastomosis was performed between the vascular pedicle and the ipsilateral facial artery and vein (Figure 3).

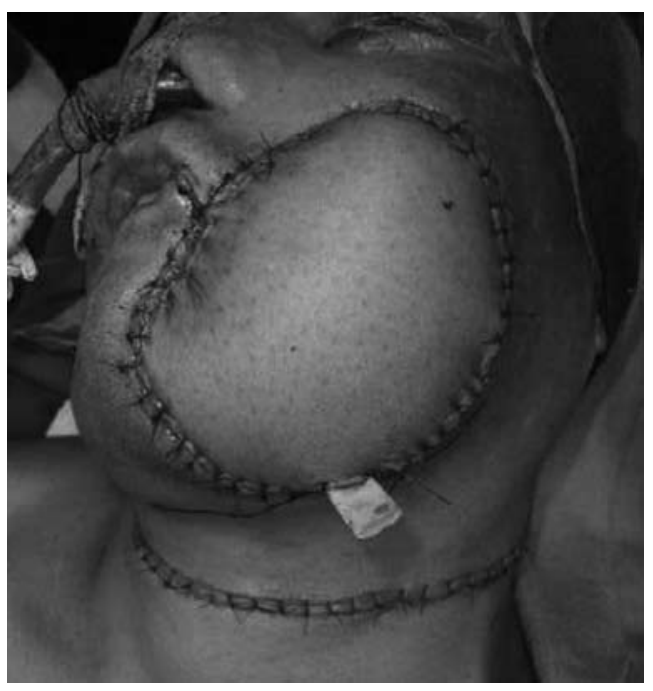

Figure 3. The ALT flap after reconstruction of the defect 


\section{CASE REPORTS}

The donor site was closed primarily. The entire operation lasted for 10 hours. The patient was extubated the following day, 12 hours after the operation. Feeding was through a nasogastric tube for 7 days until mucosal wounds were fully healed. He was discharged on the $9^{\text {th }}$ postoperative day without any complication.

Two and $1 / 2$ months post-operation, the patient was on regular diet with good oral competence. He and his family were satisfied with his appearance, although secondary operations to decrease the bulk of the flap have been recommended. Asked to rate appearance of the left thigh, his rating was "good." The patient likewise had no complaints about the function of his left thigh (Figure 4).
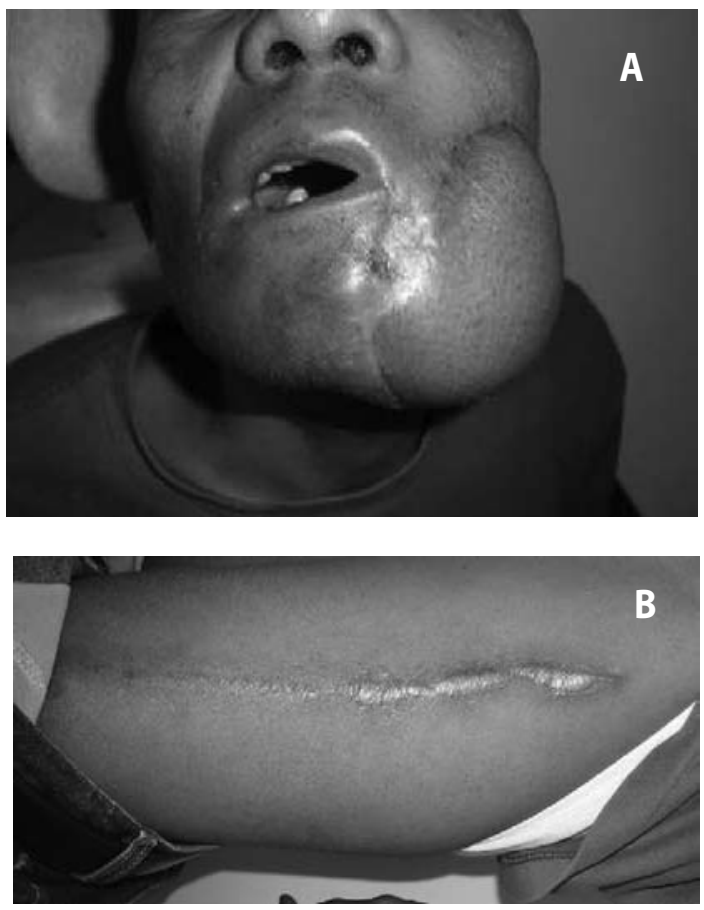

Figure 4.

A. Reconstruction $2 \frac{1}{2}$ months post-operation and adjuvant chemoradiotherapy

B. Left thigh $2 \frac{1}{2}$ months post-operation with note of only a linear scar

\section{DISCUSSION}

The advent of microvascular surgery has broadened the armamentarium of the reconstructive surgeon while eliminating problems inherent in pedicled flaps. The ALT flap, first reported in 1984 by Song ${ }^{5}$ fits the criteria of an ideal flap because it allows greater tissue yield than other flaps, allowing for folding of the flap. The operation can be carried out by two teams working simultaneously, shortening operative time. The large arterial diameter of the vascular pedicle, accompanied by two veins, facilitates easier microvascular anastomosis. The anterolateral thigh flap has good pliability and can be designed as either a single skin paddle for one layer defect reconstruction or doubleskin paddles for through-and-through defects by one perforator or multiple perforators. It can be harvested in the suprafascial plane if a thin flap is needed while the vastus lateralis and tensor fascia lata can be incorporated if bulk is required. The flap is potentially sensate by including the lateral femoral cutaneous nerve for repair. The color match is satisfactory for Orientals. The donor site can usually be closed primarily with only an inconspicuous curvilinear scar left over the thigh. ${ }^{4,5}$ It has been used successfully in the reconstruction of the laryngopharynx, oral cavity, oropharynx, external skin and maxilla. ${ }^{1}$

In our case, we harvested a $23 \times 9 \mathrm{~cm}$ flap to cover both the intraoral and skin defects. No muscle was harvested since a thin flap was needed. Although there was only 1 perforator, it was able to supply the large flap. The neck dissection was done simultaneous with flap harvesting thereby shortening total operating time. The patient's hospital stay was uneventful and he was discharged in 9 days in spite of undergoing a major operative procedure.

Donor-site morbidity was minimal. In spite of the large flap taken from the thigh, we were able to close the thigh primarily without any dehiscence or skin necrosis. As muscle harvesting was not required, there was neither loss of function nor loss of sensation. The patient rated the appearance of the thigh as good since only an easily hidden linear scar was evident on the left thigh.

The anterolateral thigh free flap is an excellent soft-tissue flap for reconstruction of a full thickness defect of the buccal area. 